\title{
Localized-density-matrix calculation of circular dichroism spectrum of optically active molecule
}

\author{
WanZhen Liang ${ }^{1}$, Satoshi Yokojima ${ }^{2}$, GuanHua Chen* \\ Department of Chemistry, The University of Hong Kong, Pokfulam Road, Hong Kong, China
}

Received 20 September 2002

\begin{abstract}
A method to calculate the circular dichroism (CD) spectra of macromolecules has been developed. The intermediate neglect of differential overlap technique for spectroscopy (INDO/S) semiempirical Hamiltonian and the full multipole expansion is adopted. The method has been employed to calculate the CD spectra of pentahelicene, and excellent agreement with the experimental result is obtained. Combined with the localized-density-matrix (LDM) method, the new method can be employed to calculate the CD spectra of large molecules.
\end{abstract}

(c) 2003 Elsevier Science B.V. All rights reserved.

\section{Introduction}

Circular dichroism (CD) is defined as the differential absorption for left and right circularly polarized lights. It provides the structural information of the optically active molecules [1-4], such as the biological chiral molecules. For instance, the CD spectra of proteins have been used to determine their secondary structures, i.e., the proportions of $\alpha$-helices, $\beta$-sheets, $\beta$ turns and random coils. Two spectral regions are of interest in the CD spectra of proteins. The far-UV $(\lambda=175-250 \mathrm{~nm})$ is dominated by contributions from the polypeptide backbone, and typically reflects the secondary structure of the protein. Many side chain chromophores exhibit CD signals in the near-UV $(\lambda=240-320 \mathrm{~nm})$ region. Both absorption and CD spectra result from the same photophysical process: the transitions of molecules from their ground states to electronically excited states. The left- and right-handedness of the CD depends on the chirality of a molecule.

Numerous works have been reported on the attempts to calculate the optical rotations or CD of proteins and other large organic molecules. These usually employ ab initio coupled-perturbed

\footnotetext{
${ }^{*}$ Corresponding author. Tel.: +852-2859-7919; fax: +852-2857-1586.

E-mail address: chemmail@hkucc.hku.hk (GH. Chen).

${ }^{1}$ Present address: Department of Chemical Engineering, University of California, Berkeley, CA 94720, USA.

${ }^{2}$ Present address: Institute of Materials Science, University of Tsukuba, 1-1-1 Ten-nodai, Tsukuba, Ibaraki 305-8573, Japan.
} 
Hartree-Fock [5,6], time-dependent density functional theory [7,8] or semiempirical $[9,10]$ calculations. The methods include the matrix method [10], the coupled oscillator model [11], the dipole interaction method, and the polarizability theory [12-14]. The matrix method assumes that a large molecule is considered to consist of $N$ separable chromophores. The total wave function is expressed as a linear superposition of basis functions $\Phi_{i a}$ which is a product of $N$ chromophores' wave functions. In the calculation, each chromophore is considered as a single unit that does not overlap or exchange electrons with other chromophores. Electronic excitations may occur within a group but not between different groups. The matrix method neglects the electron exchange between the chromophores and is a compact and efficient method for calculating the rotation strengths. The matrix method requires the parameters that describe the charge distributions associated with the different electronic states of the chromophoric groups of the protein. The parameterization is crucial to the success of this approach. Woody and coworker has improved the matrix method and has employed it to calculate the CD spectra of various secondary structures [15-18]. The coupled oscillator model accounts for intermolecular electrostatic interaction via a local field, and is effective for the molecular system that can be partitioned into two or more separate chromophores. In the dipole interaction model [9], the CD spectrum is calculated by considering individual atoms or chromophores as point dipoles. Only the dipolar interaction between the chromophores is accounted for. This treatment neglects the contributions from the higher order multipoles and the electron exchange between the chromophores. The polarizability theory [12-14] adopts the complex polarizabilities to describe the optical activity.

The CD spectra of various secondary structures, primarily helices, have been investigated by matrix method [16,17,19] and dipole interaction method [20-26]. The calculations of whole proteins have been carried out by Hirst [27] and by Woody and Sreerama [19] using the matrix method, and by Bode and Applequist [23] using the dipole interaction model. They were of reasonable success, provide the insights into the physical interpretation of the CD of given structures, and explain the reason why the CD spectra are used to determine the protein secondary structures. Recently the localized-density-matrix (LDM) method has been developed to evaluate the ground and excited state properties of very large systems [28-37]. It is based on the truncation of reduced single-electron density matrices, and thus, its computational time scales linearly with the system size. The LDM method has been generalized to include the nonorthogonal basis set [32], has been implemented with the semiempirical Hamiltonians like PPP [38], CNDO/S [39] and PM3 [40], and has subsequently been used to calculate the optical properties of polyacetylene [28-31,33,36], carbon nanotubes [33,34] and poly( $p$-phenylenevinylene) (PPV) aggregates $[35,37]$. In this work, we develop an efficient quantum mechanical method to evaluate the CD spectra of large molecular systems and their nonlocal response tensors. Although the new method can be implemented with the first-principles methods, we implemented it at semiempirical INDO/S level [41]. To test the accuracy of the new method, the CD spectrum of pentahelicene is calculated and compared to the experimental result.

\section{Formulation}

The system of our interests is subject to an electromagnetic field. To describe its dynamics under the influence of the electromagnetic field, we decompose the Lagrangian into three components:

$$
L=L_{\mathrm{mol}}+L_{\mathrm{rad}}+L_{\mathrm{int}}
$$

and under the Coulomb gauge $\nabla \cdot \mathbf{a}(\mathbf{r})=0$,

$$
L_{\mathrm{mol}}=\frac{1}{2} \sum_{\alpha} m_{\alpha} \dot{\mathbf{r}}_{\alpha}^{2}-V,
$$




$$
\begin{aligned}
& L_{\mathrm{rad}}=\frac{1}{8 \pi} \int \mathrm{d}^{3} \mathbf{r}\left\{\frac{\dot{\mathbf{a}}^{2}}{c^{2}}-(\nabla \times \mathbf{a})^{2}\right\}, \\
& L_{\mathrm{int}}=\frac{1}{c} \int \mathrm{d}^{3} \mathbf{r} \mathbf{j}^{\perp}(\mathbf{r}) \cdot \mathbf{a}(\mathbf{r}),
\end{aligned}
$$

where $m_{\alpha}$ and $\mathbf{r}_{\alpha}$ are the mass and displacement vector of the $\alpha$ th particle, respectively. $V$ is the interaction among the particles in the system, $\mathbf{a}(\mathbf{r})$ is the vector potential of electromagnetic field, and $\mathbf{j}^{\perp}(\mathbf{r})$ is the transverse part of the total current $\mathbf{j}(\mathbf{r})$,

$$
\mathbf{j}(\mathbf{r})=\sum_{\alpha} e_{\alpha} \dot{\mathbf{r}}_{\alpha} \delta\left(\mathbf{r}-\mathbf{r}_{\alpha}\right)
$$

where $e_{\alpha}$ is the charge of the particle $\alpha$.

The multipolar Lagrangian can be constructed by adding a total time derivative to the above Lagrangian:

$$
L_{\text {multi }}=L-\frac{1}{c} \frac{\mathrm{d}}{\mathrm{d} t} \int \mathrm{d}^{3} \mathbf{r} \mathbf{P}^{\perp}(\mathbf{r}) \cdot \mathbf{a}(\mathbf{r}),
$$

where $\mathbf{P}^{\perp}(\mathbf{r})$ is the transverse component of the polarization vector $\mathbf{P}(\mathbf{r})$,

$$
\mathbf{P}(\mathbf{r})=\sum_{\alpha} e_{\alpha}\left(\mathbf{r}_{\alpha}-\mathbf{R}\right) \int_{0}^{1} \mathrm{~d} \lambda \delta\left(\mathbf{r}-\mathbf{R}-\lambda\left(\mathbf{r}_{\alpha}-\mathbf{R}\right)\right) .
$$

The multipolar Hamiltonian is obtained by the canonical transformation,

$$
\hat{\mathbf{H}}_{\text {multi }}=\sum_{\alpha} \mathbf{p}_{\alpha} \cdot \dot{\mathbf{r}}_{\alpha}+\int \mathrm{d}^{3} \mathbf{r} \Pi(\mathbf{r}) \cdot \dot{\mathbf{a}}(\mathbf{r})-L_{\text {multi }},
$$

where $\mathbf{p}_{\alpha}$ and $\Pi$ are the canonical momenta conjugated to $\mathbf{r}_{\alpha}$ and $\mathbf{a}(\mathbf{r})$, respectively,

$$
\begin{aligned}
& \mathbf{p}_{\alpha}=\frac{\partial L_{\text {multi }}}{\partial \dot{\mathbf{r}}_{\alpha}}, \\
& \Pi(\mathbf{r})=\frac{\partial L_{\text {multi }}}{\partial \dot{\mathbf{a}}} .
\end{aligned}
$$

After eliminating $\dot{\mathbf{r}}_{\alpha}$ and $\dot{\mathbf{a}}$ in favour of the canonical momenta $\mathbf{p}_{\alpha}$ and $\boldsymbol{\Pi}$, we obtain the multipolar Hamiltonian

$$
\begin{aligned}
\hat{\mathbf{H}}_{\text {multi }}= & \sum_{\alpha} \frac{\mathbf{p}_{\alpha}^{2}}{2 m_{\alpha}}+V+\frac{1}{8 \pi} \int \mathrm{d}^{3} \mathbf{r}\left\{\left(\mathbf{D}^{\perp}(\mathbf{r})\right)^{2}+\mathbf{B}^{2}(\mathbf{r})\right\}-\int \mathrm{d}^{3} \mathbf{r} \mathbf{P}^{\perp}(\mathbf{r}) \cdot \mathbf{D}^{\perp}(\mathbf{r})-\int \mathrm{d}^{3} \mathbf{r} \mathbf{M}(\mathbf{r}) \cdot \mathbf{B}(\mathbf{r}) \\
& +2 \pi \int \mathrm{d}^{3} \mathbf{r}\left|\mathbf{P}^{\perp}(\mathbf{r})\right|^{2}+\sum_{\alpha} \frac{1}{2 m_{\alpha} c^{2}}\left\{\int \mathrm{d}^{3} \mathbf{r n}_{\alpha}(\mathbf{r}) \times \mathbf{B}(\mathbf{r})\right\}^{2} \\
= & \hat{\mathbf{H}}_{\mathrm{mol}}+\hat{\mathbf{H}}^{\mathrm{RAD}}+\hat{\mathbf{H}}_{\mathrm{int}}+2 \pi \int \mathrm{d}^{3} \mathbf{r}\left|\hat{\mathbf{P}}^{\perp}(\mathbf{r})\right|^{2},
\end{aligned}
$$


where

$$
\begin{aligned}
& \hat{\mathbf{H}}_{\mathrm{mol}}=\sum_{\alpha} \frac{\hat{\mathbf{p}}_{\alpha}^{2}}{2 m_{\alpha}}+\hat{V}, \\
& \hat{\mathbf{H}}^{\mathrm{RAD}}=\frac{1}{8 \pi} \int \mathrm{d}^{3} \mathbf{r}\left\{\left(\hat{\mathbf{D}}^{\perp}(\mathbf{r})\right)^{2}+\hat{\mathbf{B}}^{2}(\mathbf{r})\right\}, \\
& \hat{\mathbf{H}}_{\mathrm{int}}=-\int \mathrm{d}^{3} \mathbf{r} \hat{\mathbf{P}}^{\perp}(\mathbf{r}) \cdot \hat{\mathbf{D}}^{\perp}(\mathbf{r})-\int \mathrm{d}^{3} \mathbf{r} \hat{\mathbf{M}}(\mathbf{r}) \cdot \hat{\mathbf{B}}(\mathbf{r})+\sum_{\alpha} \frac{1}{2 m_{\alpha} c^{2}}\left\{\int \mathrm{d}^{3} \mathbf{r} \hat{\mathbf{n}}_{\alpha}(\mathbf{r}) \times \hat{\mathbf{B}}(\mathbf{r})\right\}^{2}, \\
& \hat{\mathbf{M}}(\mathbf{r})=\sum_{\alpha} \frac{1}{2 m_{\alpha}}\left\{\hat{\mathbf{n}}_{\alpha}(\mathbf{r}) \times \hat{\mathbf{p}}_{\alpha}-\hat{\mathbf{p}}_{\alpha} \times \hat{\mathbf{n}}_{\alpha}(\mathbf{r})\right\}, \\
& \hat{\mathbf{n}}_{\alpha}(\mathbf{r})=e_{\alpha}\left(\hat{\mathbf{r}}_{\alpha}-\mathbf{R}\right) \int_{0}^{1} \lambda \delta\left(\mathbf{r}-\mathbf{R}-\lambda\left(\hat{\mathbf{r}}_{\alpha}-\mathbf{R}\right)\right) \mathrm{d} \lambda, \\
& \hat{\Pi}(\mathbf{r})=\frac{\partial L_{\text {multi }}}{\partial \dot{\mathbf{a}}}=\frac{1}{4 \pi c^{2}} \dot{\hat{\mathbf{a}}}(\mathbf{r})-\frac{1}{c} \hat{\mathbf{P}}^{\perp}(\mathbf{r})=-\frac{1}{4 \pi c} \hat{\mathbf{D}}^{\perp}(\mathbf{r}), \\
& \hat{\mathbf{E}}^{\perp}(\mathbf{r})=-\frac{1}{c} \dot{\hat{\mathbf{a}}}(\mathbf{r}) .
\end{aligned}
$$

The above formalism treats the molecule as a single chromophore with its origin at $\mathbf{R}$. Complex molecules or molecular assemblies can be treated as the assemblies of interconnected chromophores, and can be divided into $N$ segments. The multipolar Hamiltonian may thus be rewritten as [42-44],

$$
\begin{aligned}
\hat{\mathbf{H}}_{\mathrm{multi}}= & \sum_{l}^{N} \hat{\mathbf{H}}_{\mathrm{mol}, l}+\sum_{k<l}^{N} \hat{\mathbf{V}}_{\mathrm{inter}}(k, l)+\frac{1}{8 \pi} \int \mathrm{d}^{3} \mathbf{r}\left(\left(\hat{\mathbf{D}}^{\perp}(\mathbf{r})\right)^{2}+\hat{\mathbf{B}}^{2}(\mathbf{r})\right)-\int \mathrm{d}^{3} \mathbf{r} \hat{\mathbf{P}}^{\perp}(\mathbf{r}) \cdot \hat{\mathbf{D}}^{\perp}(\mathbf{r}) \\
& -\int \mathrm{d}^{3} \mathbf{r} \hat{\mathbf{M}}(\mathbf{r}) \cdot \hat{\mathbf{B}}(\mathbf{r})+\sum_{l}^{N} \sum_{i \in l} \frac{1}{2 m_{i} c^{2}}\left(\int \mathrm{d}^{3} \mathbf{r} \hat{n}_{i}(l, \mathbf{r}) \times \hat{\mathbf{B}}(\mathbf{r})\right)^{2}+2 \pi \int \mathrm{d}^{3} \mathbf{r}\left|\hat{\mathbf{P}}^{\perp}(\mathbf{r})\right|^{2} \\
= & \sum_{l=1}^{N} \hat{\mathbf{H}}_{\mathrm{mol}, l}+\hat{\mathbf{V}}_{\mathrm{int}}+\hat{\mathbf{H}}^{\mathrm{RAD}}+\hat{\mathbf{H}}_{\mathrm{int}}+2 \pi \int \mathrm{d}^{3} \mathbf{r}\left|\hat{\mathbf{P}}^{\perp}(\mathbf{r})\right|^{2},
\end{aligned}
$$

where $l$ stands for the $l$ th segment, $\hat{H}_{\mathrm{mol}, l}$ is its Hamiltonian, and

$$
\hat{\mathbf{V}}_{\text {int }}=\sum_{k<l}^{N} \hat{\mathbf{V}}_{\text {inter }}(k, l)
$$

is the Coulombic intermolecular interaction between different segments. The polarization field of the entire system may be written as

$$
\hat{\mathbf{P}}(\mathbf{r})=\sum_{l} \hat{\mathbf{P}}(l, \mathbf{r})
$$

and the polarization operator of segment $l$

$$
\begin{aligned}
\hat{\mathbf{P}}(l, \mathbf{r}) & =\sum_{i \in l} e_{i}\left(\hat{\mathbf{r}}_{i}-\mathbf{R}_{l}\right) \int_{0}^{1} \mathrm{~d} \lambda \delta\left(\mathbf{r}-\mathbf{R}_{l}-\lambda\left(\hat{\mathbf{r}}_{i}-\mathbf{R}_{l}\right)\right) \\
& =\sum_{i \in l} e_{i}\left(\hat{\mathbf{r}}_{i}-\mathbf{R}_{l}\right)\left[1-\frac{1}{2 !}\left\{\left(\hat{\mathbf{r}}_{i}-\mathbf{R}_{l}\right) \cdot \vec{\nabla}\right\}+\frac{1}{3 !}\left\{\left(\hat{\mathbf{r}}_{i}-\mathbf{R}_{l}\right) \cdot \vec{\nabla}\right\}^{2}+\cdots\right] \delta\left(\mathbf{r}-\mathbf{R}_{l}\right) \\
& =\{\hat{\mu}(l)-\hat{\mathbf{Q}}(l) \cdot \vec{\nabla}+\cdots\} \delta\left(\mathbf{r}-\mathbf{R}_{l}\right),
\end{aligned}
$$

where $\hat{\mu}(l)$ and $\hat{\mathbf{Q}}(l)$ are, respectively, the dipole and quadrupole moments of the segment $l$ 


$$
\begin{aligned}
& \hat{\mu}(l)=\sum_{i \in l} e_{i}\left(\hat{\mathbf{r}}_{i}-\mathbf{R}_{l}\right), \\
& \hat{\mathbf{Q}}(l)=\frac{1}{2 !} \sum_{i \in l} e_{i}\left(\hat{\mathbf{r}}_{i}-\mathbf{R}_{l}\right)\left(\hat{\mathbf{r}}_{i}-\mathbf{R}_{l}\right)
\end{aligned}
$$

and $\mathbf{R}_{l}$ is the displacement vector for the center of segment $l ; i$ runs over all the particles in segment $l$. $\mathbf{r}_{i}$ is the displacement vector of the $i$ th particle in segment $l$.

Similarly the magnetization operator $\hat{\mathbf{M}}(\mathbf{r})$ is expressed as

$$
\hat{\mathbf{M}}(\mathbf{r})=\sum_{l} \hat{\mathbf{M}}(l, \mathbf{r})
$$

where $\hat{\mathbf{M}}(l, \mathbf{r})$ is the magnetization operator of the $l$ th molecule or segment and is expressed as

$$
\hat{\mathbf{M}}(l, \mathbf{r})=\sum_{i \in l} \frac{1}{2 m_{i} c}\left\{\hat{n}_{i}(l, \mathbf{r}) \times \hat{\mathbf{p}}_{i}-\hat{\mathbf{p}}_{i} \times \hat{n}_{i}(l, \mathbf{r})\right\},
$$

where $\mathbf{p}_{i}$ is the canonical momentum of the $i$ th electron in the $l$ th molecule or segment,

$$
\hat{\mathbf{p}}_{i}=m_{i} \dot{\mathbf{r}}_{i}-\frac{1}{c} \int\left(\hat{n}_{i}(l, \mathbf{r}) \times \hat{\mathbf{B}}(\mathbf{r})\right) \mathrm{d}^{3} \mathbf{r} .
$$

The polarization vector field operator $\hat{n}_{i}(l, \mathbf{r})$ of the $i$ th electron in the molecule $l$ is expressed as

$$
\begin{aligned}
\hat{n}_{i}(l, \mathbf{r}) & =e\left(\mathbf{r}_{i}-\mathbf{R}_{l}\right) \int_{0}^{1} \lambda \delta\left(\mathbf{r}-\mathbf{R}_{l}-\lambda\left(\mathbf{r}_{i}-\mathbf{R}_{l}\right)\right) \mathrm{d} \lambda \\
& =e\left(\mathbf{r}_{i}-\mathbf{R}_{l}\right)\left\{\frac{1}{2 !}-\frac{2}{3 !}\left(\mathbf{r}_{i}-\mathbf{R}_{l}\right) \cdot \vec{\nabla}+\frac{3}{4 !}\left(\left(\mathbf{r}_{i}-\mathbf{R}_{l}\right) \cdot \vec{\nabla}\right)^{2}+\cdots\right\} .
\end{aligned}
$$

The magnetic dipole moment of the $l$ th segment can be expressed as

$$
\hat{\mathbf{m}}(l)=\sum_{i \in l} \frac{e}{2 m_{e} c}\left(\hat{\mathbf{r}}_{i}-\mathbf{R}_{l}\right) \times \hat{\mathbf{p}}_{i} .
$$

We adopt the neoclassical approximation where the molecular system is treated quantum mechanically and the radiation field is taken to be classical obeying the Maxwell's equations. Therefore, the neoclassical Hamiltonian $H_{\text {neo }}$ can be rewritten as

$$
\begin{aligned}
\hat{\mathbf{H}}_{\mathrm{neo}}= & \sum_{l} \hat{\mathbf{H}}_{\mathrm{mol}, l}+\sum_{k<l} \hat{\mathbf{V}}_{\mathrm{int}}(k, l)-\int \mathrm{d}^{3} \mathbf{r} \hat{\mathbf{P}}(\mathbf{r}) \cdot \hat{\mathbf{D}}^{\perp}(\mathbf{r}, t)-\int \mathrm{d}^{3} \mathbf{r} \hat{\mathbf{M}}(\mathbf{r}) \cdot \hat{\mathbf{B}}(\mathbf{r}, t) \\
& +\sum_{l} \sum_{i \in l} \frac{1}{2 m_{i} c^{2}}\left(\int \mathrm{d}^{3} \mathbf{r} \hat{n}_{i}(l, \mathbf{r}) \times \hat{\mathbf{B}}(\mathbf{r}, t)\right)^{2}+2 \pi \sum_{k, l} \int \mathrm{d}^{3} \mathbf{r} \hat{\mathbf{P}}^{\perp}(l, \mathbf{r}) \cdot \hat{\mathbf{P}}^{\perp}(k, \mathbf{r}) .
\end{aligned}
$$

It can be shown that the second term on the RHS of Eq. (24) is cancelled exactly by the inter-segment contributions $(k \neq l)$ from the last term on the RHS. The Hamiltonian is thus

$$
\begin{aligned}
\hat{\mathbf{H}}_{\mathrm{neo}}= & \sum_{l} \hat{\mathbf{H}}_{\mathrm{mol}, l}-\sum_{l} \int \mathrm{d}^{3} \mathbf{r} \hat{\mathbf{P}}(l, \mathbf{r}) \cdot \hat{\mathbf{D}}^{\perp}(\mathbf{r}, t)-\sum_{l} \int \mathrm{d}^{3} \mathbf{r} \hat{\mathbf{M}}(l, \mathbf{r}) \cdot \hat{\mathbf{B}}(\mathbf{r}, t) \\
& +\sum_{l} \sum_{i \in l} \frac{1}{2 m_{i} c^{2}}\left(\int \mathrm{d}^{3} \mathbf{r} \hat{n}_{i}(l, \mathbf{r}) \times \hat{\mathbf{B}}(\mathbf{r}, t)\right)^{2}+2 \pi \sum_{l} \int \mathrm{d}^{3} \mathbf{r}\left|\hat{\mathbf{P}}^{\perp}(l, \mathbf{r})\right|^{2} .
\end{aligned}
$$

The remaining intra-segment $2 \pi \sum_{l} \int\left|\hat{\mathbf{P}}^{\perp}(l, \mathbf{r})\right|^{2} \mathrm{~d}^{3} \mathbf{r}$ accounts for the self-energy; however, it is independent of the electromagnetic field and does not play an important part in radiative processes. We neglect it in the 
calculation. In isotropic samples, such as fluids in the absence of static fields, the unweighted average of CD signal over all molecular orientations must be taken. The contribution comes from the electric dipoleelectric quadrupole term to $\mathrm{CD}$ is zero for randomly oriented systems $[43,46]$. The contribution comes from electric quadrupole and magnetic dipole is far much weaker than the interaction between the electric dipole and magnetic dipole. The CD signals in isotropic samples come mainly from the electric dipole and magnetic dipole interaction. Consequently, we expand the Hamiltonian up to the electric and magnetic dipolar terms, and neglect quadrupoles and beyond. Similarly, the fourth term on the RHS of Eq. (25) can also be omitted since it is on the order of $\mathbf{B}^{2}$. Therefore, the resulting Hamiltonian is

$$
\hat{\mathbf{H}}_{\mathrm{neo}} \approx \sum_{l} \hat{\mathbf{H}}_{\mathrm{mol}, l}-\sum_{l} \hat{\mu}(l) \cdot \hat{\mathbf{D}}^{\perp}\left(\mathbf{R}_{l}, t\right)-\sum_{l} \hat{\mathbf{m}}(l) \cdot \hat{\mathbf{B}}\left(\mathbf{R}_{l}, t\right) .
$$

Since the nuclei move much slower than the electrons, the Born-Oppenheimer approximation is employed, i.e., only the electron dynamics is considered. The equation of motion (EOM) for the reduced single-particle density matrix operator $\hat{\rho}$ is

$$
\mathrm{i} \hbar \frac{\mathrm{d} \hat{\rho}}{\mathrm{d} t}=\left[\hat{\mathbf{H}}_{\mathrm{neo}}, \hat{\rho}\right] \text {. }
$$

The EOM for the reduced single-electron density matrix $\rho \equiv\langle\hat{\rho}\rangle$ is thus

$$
\mathrm{i} \hbar \frac{\mathrm{d} \rho}{\mathrm{d} t},=[h+f+g, \rho]
$$

where $h$ is the Fock matrix, $f \equiv-\langle\hat{\mu}\rangle \cdot \mathbf{E}=-\mu \cdot \mathbf{E}$ and $g \equiv-\langle\hat{\mathbf{m}}\rangle \cdot \mathbf{B}=-\mathbf{m} \cdot \mathbf{B}$.

The CD spectroscopy measures the difference of the absorption amplitudes of a molecule for the left and right circularly polarized lights, and the CD signal can be calculated as follows:

$$
C D(\omega)=\sigma_{-}(\omega)-\sigma_{+}(\omega),
$$

where $\sigma_{-}$and $\sigma_{+}$are the absorption cross-sections of the left and right circularly polarized lights, respectively. The electric and magnetic fields of the right and left circularly polarized incident lights may be written as

$$
\begin{aligned}
& \mathbf{E}_{\text {ext }}^{ \pm}(\mathbf{r}, t)=\int \mathrm{d} \omega \hat{\mathbf{e}}_{ \pm} \mathscr{E}\left(\mathbf{k}_{ \pm}, \omega\right) \mathrm{e}^{\mathrm{i}\left(\mathbf{k}_{ \pm} \cdot \mathbf{r}-\omega t\right)}+\text { c.c. } \\
& \mathbf{B}_{\text {ext }}^{ \pm}(\mathbf{r}, t)=\int \mathrm{d} \omega \hat{\mathbf{h}}_{ \pm} \mathscr{B}\left(\mathbf{k}_{ \pm}, \omega\right) \mathrm{e}^{\mathrm{i}\left(\mathbf{k}_{ \pm} \cdot \mathbf{r}-\omega t\right)}+\text { c.c.. }
\end{aligned}
$$

$\mathbf{E}_{\mathrm{ext}}, \mathbf{B}_{\mathrm{ext}}$ and the direction of propagation of radiation $\mathbf{k}$ are mutually orthogonal. $\mathbf{E}_{\mathrm{ext}}$ and $\mathbf{B}_{\mathrm{ext}}$ are related by $\left|\mathbf{B}_{\text {ext }}\right|=n\left|\mathbf{E}_{\text {ext }}\right|$, and $n$ is the refractive index. Here the propagation vector $\mathbf{k}$ is taken along the $z$-axis.

According to the equation of the rate of energy absorption (cf. Eq. (A.11) of Appendix A) in the presence of a circularly polarized external field, the absorption cross section of the left or right circularly polarized light may be written as

$$
\begin{aligned}
\sigma_{ \pm}= & \frac{A_{ \pm}}{E_{\text {in }}} \\
= & \frac{1}{E_{\text {in }}} \int \mathrm{d}^{3} \mathbf{r} \int \mathrm{d}^{3} \mathbf{r}^{\prime} \sum_{j} 2 \omega_{j} \operatorname{Im}\left(\mathscr{E}^{*}\left(\mathbf{r}, \omega_{j}\right) \hat{\mathbf{e}}_{\mp} \cdot \alpha\left(\mathbf{r}, \mathbf{r}^{\prime}, \omega_{j}\right) \cdot \hat{\mathbf{e}}_{ \pm} \mathscr{E}\left(\mathbf{r}^{\prime}, \omega_{j}\right)\right. \\
& +\mathscr{E}^{*}\left(\mathbf{r}, \omega_{j}\right) \hat{\mathbf{e}}_{\mp} \cdot \beta\left(\mathbf{r}, \mathbf{r}^{\prime}, \omega_{j}\right) \cdot \hat{\mathbf{h}}_{ \pm} \mathscr{B}\left(\mathbf{r}^{\prime}, \omega_{j}\right)+\mathscr{B}^{*}\left(\mathbf{r}, \omega_{j}\right) \hat{\mathbf{h}}_{\mp} \cdot \gamma\left(\mathbf{r}, \mathbf{r}^{\prime}, \omega_{j}\right) \cdot \hat{\mathbf{e}}_{ \pm} \mathscr{E}\left(\mathbf{r}^{\prime}, \omega_{j}\right) \\
& \left.+\mathscr{B}^{*}\left(\mathbf{r}, \omega_{j}\right) \hat{\mathbf{h}}_{\mp} \cdot \kappa\left(\mathbf{r}, \mathbf{r}^{\prime}, \omega_{j}\right) \cdot \hat{\mathbf{h}}_{ \pm} \mathscr{B}\left(\mathbf{r}^{\prime}, \omega_{j}\right)\right)
\end{aligned}
$$


where $A_{-}\left(A_{+}\right)$is the energy absorption rate for the left (right) circularly polarized light, and

$$
E_{\text {in }}=\frac{1}{T} \int_{0}^{T} \mathrm{~d} t \frac{c}{4 \pi}\left|\mathbf{E}_{\text {ext }} \times \mathbf{H}_{\text {ext }}\right|
$$

is the energy influx of the light. $\alpha\left(\mathbf{r}, \mathbf{r}^{\prime}, \omega\right), \beta\left(\mathbf{r}, \mathbf{r}^{\prime}, \omega\right), \gamma\left(\mathbf{r}, \mathbf{r}^{\prime}, \omega\right)$, and $\kappa\left(\mathbf{r}, \mathbf{r}^{\prime}, \omega\right)$ are the nonlocal linear susceptibility tensors as defined in Eqs. (A.9) and (A.10) of Appendix A. Therefore, the CD signal at the frequency $\omega$ is expressed as

$$
\begin{aligned}
C D(\omega)= & \sigma_{-}-\sigma_{+} \\
= & \frac{1}{E_{\mathrm{in}}} \int \mathrm{d}^{3} \mathbf{r} \int \mathrm{d}^{3} \mathbf{r}^{\prime} \sum_{j} 2 \omega_{j} \operatorname{Im} \mathscr{E}^{*}\left(\mathbf{r}, \omega_{j}\right) \mathscr{B}\left(\mathbf{r}^{\prime}, \omega_{j}\right) \times\left(\frac{1}{n}\left(\hat{\mathbf{e}}_{+} \cdot \alpha\left(\mathbf{r}, \mathbf{r}^{\prime}, \omega_{j}\right) \cdot \hat{\mathbf{e}}_{-}-\hat{\mathbf{e}}_{-} \cdot \alpha\left(\mathbf{r}, \mathbf{r}^{\prime}, \omega_{j}\right) \cdot \hat{\mathbf{e}}_{+}\right)\right. \\
& +\left(\hat{\mathbf{e}}_{+} \cdot \beta\left(\mathbf{r}, \mathbf{r}^{\prime}, \omega_{j}\right) \cdot \hat{\mathbf{h}}_{-}-\hat{\mathbf{e}}_{-} \cdot \beta\left(\mathbf{r}, \mathbf{r}^{\prime}, \omega_{j}\right) \cdot \hat{\mathbf{h}}_{+}\right)+\left(\hat{\mathbf{h}}_{+} \cdot \gamma\left(\mathbf{r}, \mathbf{r}^{\prime}, \omega_{j}\right) \cdot \hat{\mathbf{e}}_{-}-\hat{\mathbf{h}}_{-} \cdot \gamma\left(\mathbf{r}, \mathbf{r}^{\prime}, \omega_{j}\right) \cdot \hat{\mathbf{e}}_{+}\right) \\
& \left.+n\left(\hat{\mathbf{h}}_{+} \cdot \kappa\left(\mathbf{r}, \mathbf{r}^{\prime}, \omega_{j}\right) \cdot \hat{\mathbf{h}}_{-}-\hat{\mathbf{h}}_{-} \cdot \kappa\left(\mathbf{r}, \mathbf{r}^{\prime}, \omega_{j}\right) \cdot \hat{\mathbf{h}}_{+}\right)\right) .
\end{aligned}
$$

For $\mathscr{E}(\mathbf{r}, \omega)=\mathscr{E}(\omega) \mathrm{e}^{\mathrm{i} \mathbf{k} \cdot \mathbf{r}}$,

$$
\begin{aligned}
C D(\omega)= & \frac{4 \pi}{c} \operatorname{Im} \int \mathrm{d}^{3} \mathbf{r} \int \mathrm{d}^{3} \mathbf{r}^{\prime} 2 \omega \mathrm{e}^{-i \mathbf{k} \cdot\left(\mathbf{r}-\mathbf{r}^{\prime}\right)}\left[\frac{1}{n}\left(\hat{\mathbf{e}}_{+} \cdot \alpha\left(\mathbf{r}, \mathbf{r}^{\prime}, \omega\right) \cdot \hat{\mathbf{e}}_{-}-\hat{\mathbf{e}}_{-} \cdot \alpha\left(\mathbf{r}, \mathbf{r}^{\prime}, \omega\right) \cdot \hat{\mathbf{e}}_{+}\right)\right. \\
& +\left(\hat{\mathbf{e}}_{+} \cdot \beta\left(\mathbf{r}, \mathbf{r}^{\prime}, \omega\right) \cdot \hat{\mathbf{h}}_{-}-\hat{\mathbf{e}}_{-} \cdot \beta\left(\mathbf{r}, \mathbf{r}^{\prime}, \omega\right) \cdot \hat{\mathbf{h}}_{+}\right)+\left(\hat{\mathbf{h}}_{+} \cdot \gamma\left(\mathbf{r}, \mathbf{r}^{\prime}, \omega\right) \cdot \hat{\mathbf{e}}_{-}-\hat{\mathbf{h}}_{-} \cdot \gamma\left(\mathbf{r}, \mathbf{r}^{\prime}, \omega\right) \cdot \hat{\mathbf{e}}_{+}\right) \\
& \left.+n\left(\hat{\mathbf{h}}_{+} \cdot \kappa\left(\mathbf{r}, \mathbf{r}^{\prime}, \omega\right) \cdot \hat{\mathbf{h}}_{-}-\hat{\mathbf{h}}_{-} \cdot \kappa\left(\mathbf{r}, \mathbf{r}^{\prime}, \omega\right) \cdot \hat{\mathbf{h}}_{+}\right)\right]
\end{aligned}
$$

We note that the $\mathrm{CD}$ signals are determined once the linear susceptibility tensors $\alpha, \beta, \gamma$ and $\kappa$, are known $[13,14]$. The detailed expressions of these terms are given in Appendix A.

\section{Implementation}

In this work, we treat our molecule as single segment. Since the computational cost in the time-domain is far much less than that in the frequency domain, we integrate the EOM in the time-domain. To evaluate the molecular polarization $\mathscr{P}(\mathbf{r}, t)$ and magnetization $\mathscr{M}(\mathbf{r}, t)$, we need to obtain the reduced single-electron density matrix $\rho(t)$ of the system. We have shown how to obtain $\rho(t)$ in the previous papers [28-33,35]. The long wavelength limit has been employed and the spatial variation of the amplitudes of the external electromagnetic field over the system have been neglected in the previous calculations for a normal linear absorption. Unless the size of molecular system of interest is comparable to the light wavelength, spatial variation of $E_{\text {ext }}$ and $B_{\text {ext }}$ can be neglected in the calculation. In another word, the electromagnetic field is assumed to be uniform over the extent of molecule. The electric and magnetic response tensors $\alpha\left(\mathbf{r}, \mathbf{r}^{\prime}, \omega\right)$, $\beta\left(\mathbf{r}, \mathbf{r}^{\prime}, \omega\right), \gamma\left(\mathbf{r}, \mathbf{r}^{\prime}, \omega\right)$ and $\kappa\left(\mathbf{r}, \mathbf{r}^{\prime}, \omega\right)$ are thus uniform over the molecule, i.e., they do not depend on $\mathbf{r}$ and $\mathbf{r}^{\prime}$ : $\alpha\left(\mathbf{r}, \mathbf{r}^{\prime}, \omega\right)=\alpha(\omega), \beta\left(\mathbf{r}, \mathbf{r}^{\prime}, \omega\right)=\beta(\omega), \gamma\left(\mathbf{r}, \mathbf{r}^{\prime}, \omega\right)=\gamma(\omega)$, and $\kappa\left(\mathbf{r}, \mathbf{r}^{\prime}, \omega\right)=\kappa(\omega)$. Furthermore, the first and last terms in Eq. (34) are zero. Therefore,

$$
C D(\omega)=\frac{4 \pi}{c} \operatorname{Im} \int \mathrm{d}^{3} \mathbf{r} \int \mathrm{d}^{3} \mathbf{r}^{\prime} 2 \omega \mathrm{e}^{-i \mathbf{k} \cdot\left(\mathbf{r}-\mathbf{r}^{\prime}\right)}\left[\left(\hat{\mathbf{e}}_{+} \cdot \beta(\omega) \cdot \hat{\mathbf{h}}_{-}-\hat{\mathbf{e}}_{-} \cdot \beta(\omega) \cdot \hat{\mathbf{h}}_{+}\right)+\left(\hat{\mathbf{h}}_{+} \cdot \gamma(\omega) \cdot \hat{\mathbf{e}}_{-}-\hat{\mathbf{h}}_{-} \cdot \gamma(\omega) \cdot \hat{\mathbf{e}}_{+}\right)\right],
$$

and we need only to calculate $\beta(\omega)$ and $\gamma(\omega)$. 
To evaluate $\beta(\omega)$ and $\gamma(\omega)$, we need to calculate the linear response of electrons subject to the external electromagnetic field. $\delta \rho^{(1)}$, the linear response of the optical field, is calculated by solving the time-dependent Hartree-Fock (TDHF) equation of motion

$$
\mathrm{i}\left(\hbar \frac{\mathrm{d}}{\mathrm{d} t}+\gamma\right) \delta \rho^{(1)}=\left[\delta h^{(1)}, \rho^{(0)}\right]+\left[h^{(0)}, \delta \rho^{(1)}(t)\right]+\left[f, \rho^{(0)}\right]+\left[g, \rho^{(0)}\right] .
$$

Here $h^{(0)}$ is the ground state Fock matrix, $\delta h^{(1)}$ is the first-order induced Fock matrix, $f(g)$ describes the interaction between the electrons and the external electric (magnetic) field and $\gamma$ is the phenomenological dephasing constant. The fourth-order Runge-Kutta method [47] is employed to integrate Eq. (36). The molecular polarization $\mathbf{P}(t)$ and magnetization $\mathbf{M}(t)$ can be evaluated after $\delta \rho^{(1)}$ is known. Then the response tensors are readily to be obtained. From Eqs. (A.22)-(A.25), we note that $\gamma(\omega)$ is related only to the interaction term between the system and the external electric field while $\beta(\omega)$ is only related to the interaction term between the system and the external magnetic field. Thus, to calculate $\gamma(\omega)$, we assume that the system interacts only with the external electric field $\mathscr{E}$. The magnetization $\mathbf{M}(t)$ is evaluated in time-domain and the Fourier transformation is performed to obtain $\mathbf{M}(\omega), \gamma(\omega)$ is related to $\mathbf{M}(\omega)$ by

$$
\gamma_{i j}(\omega)=\left.\frac{\mathrm{d}_{i}(\omega)}{\mathrm{d} \mathscr{E}_{j}(\omega)}\right|_{\mathscr{E}=0}
$$

To calculate $\beta(\omega)$, we assume the system interacts only with the external magnetic field $\mathscr{B}$. $\mathbf{P}$ is then evaluated. $\beta(\omega)$ is related to $\mathbf{P}$ by

$$
\beta_{i j}(\omega)=\left.\frac{\mathrm{d} \mathbf{P}_{i}(\omega)}{\mathrm{d} \mathscr{B}_{j}(\omega)}\right|_{\mathscr{B}=0}
$$

Once we know $\beta(\omega)$ and $\gamma(\omega)$, the CD signal is thus readily obtained.

One thorny issue in evaluating the CD spectrum is the origin dependence of the magnetic dipole element $\mathbf{m}$ which leads often to the origin dependence of CD signal. Many proposals to eliminate this dependence have been proposed, one of which is employing the origin-independent atomic orbitals [48]. We propose here a method to ensure the origin invariance [3]. Rewriting Eq. (23) we obtain that

$$
\hat{\mathbf{m}}=\sum_{i} \frac{e}{2 c m} \hat{\mathbf{r}}_{i} \times \hat{\mathbf{p}}_{i}-\frac{e}{2 c m} \mathbf{R} \times \hat{\mathbf{p}}
$$

where $\hat{\mathbf{p}}$ is the total momentum of the electrons. If the origin shifts $\Delta \mathbf{R}$, the magnetic moment changes to $\mathbf{m}^{\prime}=\mathbf{m}+(e / 2 \mathrm{~cm}) \Delta \mathbf{R} \times \mathbf{p}$. To ensure the origin invariance, we use the following commutator relation to calculate the momentum of individual electron:

$$
\hat{\mathbf{p}}_{i}=-\frac{m_{e}}{\mathrm{i} \hbar}\left[\hat{\mathbf{H}}, \hat{\mathbf{r}}_{i}\right] \text {. }
$$

Therefore, it can be shown that the total momentum $\mathbf{p}$ is proportional to the total transition dipole momentum $\boldsymbol{\mu}$, i.e.,

$$
\mathbf{p}_{k l}=-\frac{m_{e}}{\mathrm{i} e \hbar}\left(\epsilon_{k}-\epsilon_{l}\right) \boldsymbol{\mu}_{k l},
$$

where $k(l)$ stands for the eigenstate $|k\rangle(|l\rangle)$ of Hamiltonian $\hat{H}$ with corresponding energy $\epsilon_{k}\left(\epsilon_{l}\right)$, and $\mathbf{p}_{k l}$ $\left(\boldsymbol{\mu}_{k l}\right)$ is the matrix element of $\mathbf{p}(\boldsymbol{\mu})$ between $|k\rangle$ and $|l\rangle$. This leads to

$$
\boldsymbol{\mu}_{l k} \cdot\left(\Delta \mathbf{R} \times \mathbf{p}_{k l}\right)=0 .
$$

This eliminates thus the origin dependence of the calculated CD signals. 


\section{CD spectrum of pentahelicene}

The helicenes are model molecules for screw-shaped molecular systems which are important in nucleic acid, peptide and sugar chemistry. They exhibit CDs and optical rotations. We choose pentahelicenes to test our formalism by calculating its $\mathrm{CD}$ spectrum and compare the resulting spectrum to the experimental result. Experimental CD as well as absorption and fluorescence spectra [50,51] have been measured for pentahelicene. TDDFT calculation of its CD spectrum has been carried out [7], and the calculated result was consistent with the experiments. In our calculation, we employ the semiempirical INDO/S Hamiltonian. The Coulomb interaction among electrons is explicitly taken into account, and the electron-electron correlation is accounted for within the TDHF approximation as described in Sections 2 and 3 .

The geometry of pentahelicene is taken from the supporting information of [7]. Its structure is shown in the inset of Fig. 1(a). The calculated absorption spectrum is shown in Fig. 1(a). Compared to the experimental data, the overall shape agrees well, and however, the calculated energies of the excited states are slightly larger. To be consistent with the measured spectra, we red-shift the calculated absorption and the subsequent CD spectra by $0.22 \mathrm{eV}$. We solved Eq. (28) for $\delta \rho^{(1)}$ and evaluated the CD

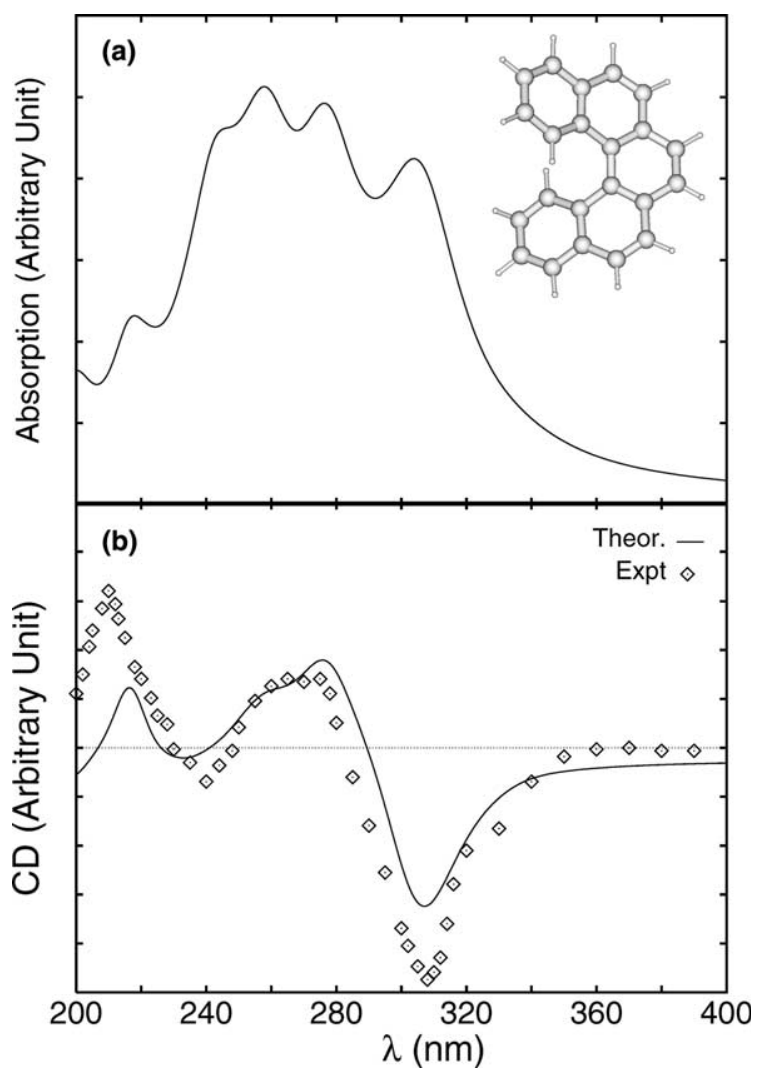

Fig. 1. The calculated and experimental absorption and CD spectra of pentahelicene. (a) Absorption spectrum; (b) the solid line is simulated by full TDHF and the diamond is experimental result taken from [7]. The calculated absorption and CD spectra are redshifted by $0.22 \mathrm{eV}$. 
spectrum according the steps described in Sections 2 and 3. The experiment was carried on pentahelicene in solution, and the measured spectral properties are the averaged result for random orientations. To compare the calculated result with the experimental data, the CD spectrum needs to be averaged over $x, y$ and $z$ directions, i.e., $C D(\omega)=\frac{1}{3}\left(C D_{x}(\omega)+C D_{y}(\omega)+C D_{z}(\omega)\right)$. In the calculation, we applied in turn the electromagnetic field propagating along $x$-, $y$ - and $z$-axis. The corresponding $\mathrm{CD}$ spectra in these three directions were evaluated, and the orientation averaging of $\mathrm{CD}$ signals was taken to obtain the calculated CD spectrum in Fig. 1(b). The experimental CD [7] is plotted in Fig. 1 for comparison. The amplitude of the calculated CD spectrum is arbitrarily scaled to fit the experimental amplitude. Encouraging agreement between the observed and calculated results are obtained. The characteristic positive band at long wavelength and the negative band at short wavelength reasonably agree with the experimental results.

\section{Discussion}

We propose a new formalism to evaluate CD spectrum within the TDHF approximation. Instead of solving for many-electron wave function, the reduced single-electron density matrix $\rho$ is determined by integrating its EOM in the time-domain. The consistence between the calculated and measured pentahelicene CD spectra validates the new formalism. The new formalism is quite different from other existing methods. The rotatory strength is a central quantity describing the optical activity. To determine the CD spectrum or optical activity, the rotatory strengths $R_{0 f}=\operatorname{Im}\langle 0|\mu| f\rangle\langle f|m| 0\rangle$ for transitions from ground state $|0\rangle$ to excited states $|f\rangle$ s needs to be determined. This is achieved by solving for many-body wave function. The CD signal exists only when the electric and magnetic dipole transitions induced by the external electric and magnetic field are simultaneously allowed (the second and third term in our Eq. (34). In our formalism, we evaluate the linear response tensors (see Eq. (34)) which are calculated directly from the reduced single-electron density matrix. Since many-body wave function is not required, our formalism is potentially very efficient for large molecular systems.

In this work, our formalism is implemented at the semiempirical INDO/S level. However, it can be generalized easily for ab initio molecular orbital calculations or time-dependent density functional theory (TDDFT). Like other methods, the long wavelength approximation is currently adopted in our calculation. This leads to the elimination of first and fourth terms in Eq. (34). It is desirable to incorporate the spatial dependence of the electromagnetic field when the sizes of the molecular systems of interests are not negligible compared to the wavelength. This can be achieved simply by retaining the spatial dependence of the electromagnetic field in Eq. (28). Compared to the existing methods, our formalism treats quantum mechanically the entire molecule as a whole and may thus require quite large amount of computational resources. The solution is to combine our formalism with efficient quantum chemistry methods. One possible way is incorporating our formalism within the general framework of the LDM method. The LDM method is a linear-scaling quantum mechanical method for ground and excited states. We expect thus that our new formalism can be used to calculate the CD or optical activities of very large chiral molecular systems, such as protein.

\section{Acknowledgements}

We thank Dr. XiuJun Wang for reading thoroughly the manuscript and constructive comments. Support from the Hong Kong Research Grant Council (RGC) and the Committee for Research and Conference Grants (CRCG) of the University of Hong Kong is gratefully acknowledged. 


\section{Appendix A. The energy absorption rate and response tensors}

The rate of energy absorption $A(t)$ by the system can be represented as [14,44]

$$
\begin{aligned}
A(t) & =\frac{1}{T} \int_{0}^{T} \mathrm{~d} t \frac{\mathrm{d}\left\langle\hat{H}_{\text {neo }}\right\rangle}{\mathrm{d} t}=\frac{1}{T} \int_{0}^{T} \mathrm{~d} t\left\{\operatorname{Tr}\left[\frac{\mathrm{d} \hat{H}_{\text {neo }}}{\mathrm{d} t} \rho\right]+\operatorname{Tr}\left[\frac{\mathrm{d} \rho}{\mathrm{d} t} \hat{H}_{\text {neo }}\right]\right\} \\
& =-\frac{1}{T} \int_{0}^{T} \mathrm{~d} t\left\{\int \mathrm{d}^{3} \mathbf{r} \frac{\partial \mathbf{E}^{\perp}(\mathbf{r}, t)}{\partial t} \cdot\langle\hat{\mathbf{P}}(\mathbf{r})\rangle+\int \mathrm{d}^{3} \mathbf{r} \frac{\partial \mathbf{B}^{\perp}(\mathbf{r}, t)}{\partial t} \cdot\langle\hat{\mathbf{M}}(\mathbf{r})\rangle\right\} \\
& =-\frac{1}{T} \int_{0}^{T} \mathrm{~d} t\left\{\int \mathrm{d}^{3} \mathbf{r} \frac{\partial \mathbf{E}^{\perp}(\mathbf{r}, t)}{\partial t} \cdot \mathbf{P}(\mathbf{r}, t)+\int \mathrm{d}^{3} \mathbf{r} \frac{\partial \mathbf{B}^{\perp}(\mathbf{r}, t)}{\partial t} \cdot \mathbf{M}(\mathbf{r}, t)\right\} .
\end{aligned}
$$

The time-dependent density operator $\rho$, polarization $\mathbf{P}(\mathbf{r}, t)$ and magnetization $\mathbf{M}(\mathbf{r}, t)$ can be expanded in powers of the radiation field, i.e.,

$$
\begin{aligned}
& \rho(t)=\rho^{(0)}+\delta \rho^{(1)}(t)+\delta \rho^{(2)}(t)+\cdots, \\
& \mathbf{P}(\mathbf{r}, t)=\mathbf{P}^{(0)}(\mathbf{r})+\mathbf{P}^{(1)}(\mathbf{r}, t)+\mathbf{P}^{(2)}(\mathbf{r}, t)+\cdots, \\
& \mathbf{M}(\mathbf{r}, t)=\mathbf{M}^{(0)}(\mathbf{r})+\mathbf{M}^{(1)}(\mathbf{r}, t)+\mathbf{M}^{(2)}(\mathbf{r}, t)+\cdots .
\end{aligned}
$$

Here $\delta \rho^{(n)}, \mathbf{P}^{(n)}$ and $\mathbf{M}^{(n)}$ are $n$th order $(n>1)$ induced density operator, polarization and magnetization. The $n$th order induced polarization and magnetization will be expanded in the form

$$
\begin{aligned}
& \mathbf{P}^{(n)}(\mathbf{r}, t)=\sum_{s} \mathbf{P}^{(n)}\left(\mathbf{r}, \omega_{s}\right) \mathrm{e}^{-\mathrm{i} \omega_{s} t}+\text { c.c. }, \\
& \mathbf{M}^{(n)}(\mathbf{r}, t)=\sum_{s} \mathbf{M}^{(n)}\left(\mathbf{r}, \omega_{s}\right) \mathrm{e}^{-\mathrm{i} \omega_{s} t}+\text { c.c. }
\end{aligned}
$$

$\omega_{s}$ is any combination of the incoming frequencies $\omega_{s}= \pm \omega_{1} \pm \omega_{2} \pm \omega_{3} \pm \cdots \pm \omega_{n}$. Then $A(t)$ can also be expended as $A(t)=A^{(1)}(t)+A^{(2)}(t)+\cdots$. Hereafter we will only concern with linear absorptions. The higher order terms of $A(t)$, which contain the effects of multipole scattering in the sample, are neglected. The rate of the energy absorption is expressed as

$$
\begin{aligned}
A(t)= & -\frac{1}{T} \int_{0}^{T} \mathrm{~d} t\left\{\int \mathrm{d}^{3} \mathbf{r} \frac{\partial \mathbf{E}_{\text {ext }}(\mathbf{r}, t)}{\partial t} \cdot \mathbf{P}^{(1)}(\mathbf{r}, t)+\int \mathrm{d}^{3} \mathbf{r} \frac{\partial \mathbf{B}_{\text {ext }}(\mathbf{r}, t)}{\partial t} \cdot \mathbf{M}^{(1)}(\mathbf{r}, t)\right. \\
& +\int \mathrm{d}^{3} \mathbf{r} \frac{\partial \mathbf{E}^{\prime}(1)(\mathbf{r}, t)}{\partial t} \cdot \mathbf{P}^{(1)}(\mathbf{r}, t)+\int \mathrm{d}^{3} \mathbf{r} \frac{\partial \mathbf{B}^{\prime}(1)(\mathbf{r}, t)}{\partial t} \cdot \mathbf{M}^{(1)}(\mathbf{r}, t)+\int \mathrm{d}^{3} \mathbf{r} \frac{\partial \mathbf{E}_{\text {ext }}(\mathbf{r}, t)}{\partial t} \cdot \mathbf{P}^{(0)}(\mathbf{r}) \\
& \left.+\int \mathrm{d}^{3} \mathbf{r} \frac{\partial \mathbf{E}^{\prime}(1)(\mathbf{r}, t)}{\partial t} \cdot \mathbf{P}^{(0)}(\mathbf{r})\right\} .
\end{aligned}
$$

The last two terms are zero when we take the average of Eq. (A.7) over an optical cycle. The Maxwell transverse vector electric field and magnetic field have been partitioned into two parts as

$$
\hat{\mathbf{E}}^{\perp}(\mathbf{r}, t)=\hat{\mathbf{E}}_{\mathrm{ext}}(\mathbf{r}, t)+\hat{\mathbf{E}}^{\prime}(\mathbf{r}, t), \quad \hat{\mathbf{B}}^{\perp}(\mathbf{r}, t)=\hat{\mathbf{B}}_{\mathrm{ext}}(\mathbf{r}, t)+\hat{\mathbf{B}}^{\prime}(\mathbf{r}, t) .
$$

The primed fields are the induced fields due to the multipole scattering of the incoming wave by the charge distribution and can be expressed in terms of retarded Green's functions [45]. Then Eq. (A.7) will be changed to 


$$
\begin{aligned}
A= & -\sum_{j} \int \mathrm{d}^{3} \mathbf{r}\left[-\mathrm{i} \omega_{j} \mathscr{E}\left(\mathbf{r}, \omega_{j}\right) \cdot \mathscr{P}^{*^{(1)}}\left(\mathbf{r}, \omega_{j}\right)+\mathrm{i} \omega_{j} \mathscr{E}^{*}\left(\mathbf{r}, \omega_{j}\right) \cdot \mathscr{P}^{(1)}\left(\mathbf{r}, \omega_{j}\right)\right. \\
& \left.-\mathrm{i} \omega_{j} \mathscr{B}\left(\mathbf{r}, \omega_{j}\right) \cdot \mathscr{M}^{*^{(1)}}\left(\mathbf{r}, \omega_{j}\right)+\mathrm{i} \omega_{j} \mathscr{B}^{*}\left(\mathbf{r}, \omega_{j}\right) \cdot \mathscr{M}^{(1)}\left(\mathbf{r}, \omega_{j}\right)\right] .
\end{aligned}
$$

The average of Eq. (A.8) over an optical cycle has been made. We also have assumed that the probe field envelop varies slowly in time compared with its optical period. The induced linear polarization and magnetization may be given by

$$
\begin{aligned}
& \mathscr{P}^{(1)}(\mathbf{r}, \omega)=\int \mathrm{d}^{3} \mathbf{r}^{\prime} \alpha\left(\mathbf{r}, \mathbf{r}^{\prime}, \omega\right) \cdot \mathscr{E}\left(\mathbf{r}^{\prime}, \omega\right)+\int \mathrm{d}^{3} \mathbf{r}^{\prime} \beta\left(\mathbf{r}, \mathbf{r}^{\prime}, \omega\right) \cdot \mathscr{B}\left(\mathbf{r}^{\prime}, \omega\right), \\
& \mathscr{M}^{(1)}(\mathbf{r}, \omega)=\int \mathrm{d}^{3} \mathbf{r}^{\prime} \gamma\left(\mathbf{r}, \mathbf{r}^{\prime}, \omega\right) \cdot \mathscr{E}\left(\mathbf{r}^{\prime}, \omega\right)+\int \mathrm{d}^{3} \mathbf{r}^{\prime} \kappa\left(\mathbf{r}, \mathbf{r}^{\prime}, \omega\right) \cdot \mathscr{B}\left(\mathbf{r}^{\prime}, \omega\right) .
\end{aligned}
$$

$\alpha, \beta, \gamma$ and $\kappa$ are the nonlocal response tensors which describe all linear optical phenomena. Substituting Eqs. (A.9) and (A.10) into Eq. (A.8), we have

$$
\begin{aligned}
A= & -\int \mathrm{d}^{3} \mathbf{r} \int \mathrm{d}^{3} \mathbf{r}^{\prime} \sum_{j}\left[-\mathrm{i} \omega_{j}\left(\mathscr{E}\left(\mathbf{r}, \omega_{j}\right) \cdot \alpha^{*}\left(\mathbf{r}, \mathbf{r}^{\prime}, \omega_{j}\right) \cdot \mathscr{E}^{*}\left(\mathbf{r}^{\prime}, \omega_{j}\right)\right.\right. \\
& +\mathscr{E}\left(\mathbf{r}, \omega_{j}\right) \cdot \beta^{*}\left(\mathbf{r}, \mathbf{r}^{\prime}, \omega_{j}\right) \cdot \mathscr{B}^{*}\left(\mathbf{r}^{\prime}, \omega_{j}\right)+\mathscr{B}\left(\mathbf{r}, \omega_{j}\right) \cdot \gamma^{*}\left(\mathbf{r}, \mathbf{r}^{\prime}, \omega_{j}\right) \cdot \mathscr{E}^{*}\left(\mathbf{r}^{\prime}, \omega_{j}\right) \\
& \left.+\mathscr{B}\left(\mathbf{r}, \omega_{j}\right) \cdot \kappa^{*}\left(\mathbf{r}, \mathbf{r}^{\prime}, \omega_{j}\right) \cdot \mathscr{B}^{*}\left(\mathbf{r}^{\prime}, \omega_{j}\right)\right)+\mathrm{i} \omega_{j}\left(\mathscr{E}^{*}\left(\mathbf{r}, \omega_{j}\right) \cdot \alpha\left(\mathbf{r}, \mathbf{r}^{\prime}, \omega_{j}\right) \cdot \mathscr{E}\left(\mathbf{r}^{\prime}, \omega_{j}\right)\right. \\
& +\mathscr{E}^{*}\left(\mathbf{r}, \omega_{j}\right) \cdot \beta\left(\mathbf{r}, \mathbf{r}^{\prime}, \omega_{j}\right) \cdot \mathscr{B}\left(\mathbf{r}^{\prime}, \omega_{j}\right)+\mathscr{B}^{*}\left(\mathbf{r}, \omega_{j}\right) \cdot \gamma\left(\mathbf{r}, \mathbf{r}^{\prime}, \omega_{j}\right) \cdot \mathscr{E}\left(\mathbf{r}^{\prime}, \omega_{j}\right) \\
& \left.\left.+\mathscr{B}^{*}\left(\mathbf{r}, \omega_{j}\right) \cdot \kappa\left(\mathbf{r}, \mathbf{r}^{\prime}, \omega_{j}\right) \cdot \mathscr{B}\left(\mathbf{r}^{\prime}, \omega_{j}\right)\right)\right] \\
= & \int \mathrm{d}^{3} \mathbf{r} \int \mathrm{d}^{3} \mathbf{r}^{\prime} \sum_{j} 2 \omega_{j} \operatorname{Im}\left(\mathscr{E}\left(\mathbf{r}, \omega_{j}\right) \cdot \alpha\left(\mathbf{r}, \mathbf{r}^{\prime}, \omega_{j}\right) \cdot \mathscr{E}\left(\mathbf{r}^{\prime}, \omega_{j}\right)+\mathscr{E}\left(\mathbf{r}, \omega_{j}\right) \cdot \beta\left(\mathbf{r}, \mathbf{r}^{\prime}, \omega_{j}\right) \cdot \mathscr{B}\left(\mathbf{r}^{\prime}, \omega_{j}\right)\right. \\
& \left.+\mathscr{B}^{*}\left(\mathbf{r}, \omega_{j}\right) \cdot \gamma\left(\mathbf{r}, \mathbf{r}^{\prime}, \omega_{j}\right) \cdot \mathscr{E}\left(\mathbf{r}^{\prime}, \omega_{j}\right)+\mathscr{B}\left(\mathbf{r}, \omega_{j}\right) \cdot \kappa\left(\mathbf{r}, \mathbf{r}^{\prime}, \omega_{j}\right) \cdot \mathscr{B}\left(\mathbf{r}^{\prime}, \omega_{j}\right)\right) .
\end{aligned}
$$

That is, the rate of energy absorption $A$ is proportional to the imaginary part of the linear susceptibility $\alpha, \beta$, $\gamma$ and $\kappa$. These response tensors can be obtained from $\mathbf{P}(\mathbf{r}, t)$ and $\mathbf{M}(\mathbf{r}, t)$. Therefore the central problem in the fs pump-probe experiment lies in the calculation of $\mathbf{P}(\mathbf{r}, t)$ and $\mathbf{M}(\mathbf{r}, t)$, i.e.,

$$
\begin{aligned}
& \mathbf{P}(\mathbf{r}, t)=\langle\Phi(\mathbf{r}, t)|\hat{\mathbf{P}}(\mathbf{r})| \Phi(\mathbf{r}, t)\rangle=\operatorname{Tr}(\rho \hat{\mathbf{P}}(\mathbf{r})), \\
& \mathbf{M}(\mathbf{r}, t)=\langle\Phi(\mathbf{r}, t)|\hat{\mathbf{M}}(\mathbf{r})| \Phi(\mathbf{r}, t)\rangle=\operatorname{Tr}(\rho \hat{\mathbf{M}}(\mathbf{r})) .
\end{aligned}
$$

Using the Liouville space notation [44], the density $\rho(t)$ can be written as

$$
\mathrm{i}\left(\hbar \frac{\mathrm{d}}{\mathrm{d} t}+\gamma\right) \rho(t)=\hat{L} \rho(t)+\hat{L}_{\text {int }} \rho(t) .
$$

$\hat{L}$ and $\hat{L}_{\text {int }}$ are Liouville space operators defined by their actions on an operator $A$, that is

$$
\hat{L} \hat{A}=[\hat{H}, \hat{A}], \quad \hat{L}_{\text {int }} \hat{A}=\left[\hat{H}_{\text {int }}, \hat{A}\right] .
$$

The first-order density matrix can be expressed as [44]

$$
\begin{aligned}
\delta \rho^{(1)}(t) & =-\frac{\mathrm{i}}{\hbar} \int_{t_{0}}^{t} \mathrm{~d} t^{\prime} \theta\left(t-t^{\prime}\right) \exp \left(\left(-\mathrm{i} \frac{\hat{L}}{\hbar}-\frac{\gamma}{\hbar}\right)\left(t-t^{\prime}\right)\right) \hat{L}_{\text {int }}\left(t^{\prime}\right) \times \theta\left(t^{\prime}-t_{0}\right) \exp \left(\left(-\mathrm{i} \frac{\hat{L}}{\hbar}-\frac{\gamma}{\hbar}\right)\left(t^{\prime}-t_{0}\right)\right) \rho\left(t_{0}\right) \\
& =-\frac{\mathrm{i}}{\hbar} \int_{0}^{\infty} \mathrm{d} t_{1} \theta\left(t_{1}\right) \exp \left(\left(-\mathrm{i} \frac{\hat{L}}{\hbar}-\frac{\gamma}{\hbar}\right) t_{1}\right) \hat{L}_{\text {int }}\left(t-t_{1}\right) \rho^{(0)} .
\end{aligned}
$$


Since $\rho\left(t_{0}\right)$ represents the equilibrium density operator, it does not evolve with time when subject to the material Hamiltonian with no field. This implies

$$
\theta\left(t^{\prime}-t_{0}\right) \exp \left(\left(-\mathrm{i} \frac{\hat{L}}{\hbar}-\frac{\gamma}{\hbar}\right)\left(t^{\prime}-t_{0}\right)\right) \rho\left(t_{0}\right)=\rho\left(t_{0}\right) .
$$

Here $\theta(t)$ is Heavyside step function $[\theta(t)=1$ for $t>0, \theta(t)=0$ for $t<0]$. The first-order polarization $\mathbf{P}^{(1)}(\mathbf{r}, t)$ and magnetization $\mathbf{M}^{(1)}(\mathbf{r}, t)$ take the forms

$$
\begin{aligned}
\mathbf{P}^{(1)}(\mathbf{r}, t)= & \operatorname{Tr}\left[\hat{\mathbf{P}}(\mathbf{r}) \delta \rho^{(1)}(t)\right] \\
= & \operatorname{Tr}\left[\hat{\mathbf{P}}(\mathbf{r})\left(-\frac{\mathrm{i}}{\hbar} \int_{0}^{\infty} \mathrm{d} t_{1} \theta\left(t_{1}\right) \exp \left(\left(-\mathrm{i} \frac{\hat{L}}{\hbar}-\frac{\gamma}{\hbar}\right) t_{1}\right) L_{\mathrm{int}}\left(t-t_{1}\right) \rho^{(0)}\right)\right] \\
= & \operatorname{Tr}\left\{\hat{\mathbf{P}}(\mathbf{r}) \frac{\mathrm{i}}{\hbar} \int_{0}^{\infty} \mathrm{d} t_{1} \theta\left(t_{1}\right) \exp \left(\left(-\mathrm{i} \frac{\hat{L}}{\hbar}-\frac{\gamma}{\hbar}\right) t_{1}\right)\right. \\
& \left.\times\left[\int \mathrm{d}^{3} \mathbf{r}^{\prime} \hat{\mathbf{P}}\left(\mathbf{r}^{\prime}\right) \cdot \mathbf{E}\left(\mathbf{r}^{\prime}, t-t_{1}\right)+\int \mathrm{d}^{3} \mathbf{r}^{\prime} \hat{\mathbf{M}}\left(\mathbf{r}^{\prime}\right) \cdot \mathbf{B}\left(\mathbf{r}^{\prime}, t-t_{1}\right), \rho^{(0)}\right]\right\}, \\
\mathbf{M}^{(1)}(\mathbf{r}, t)= & \operatorname{Tr}\left[\hat{\mathbf{M}}(\mathbf{r}) \delta \rho^{(1)}(t)\right] \\
= & \operatorname{Tr}\left[\hat{\mathbf{M}}(\mathbf{r})\left(-\frac{1}{\hbar} \int_{0}^{\infty} \mathrm{d} t_{1} \theta\left(t_{1}\right) \exp \left(\left(-\mathrm{i} \frac{\hat{L}}{\hbar}-\frac{\gamma}{\hbar}\right) t_{1}\right) L_{\text {int }}\left(t-t_{1}\right) \rho^{(0)}\right)\right] \\
= & \operatorname{Tr}\left\{\hat{\mathbf{M}}(\mathbf{r}) \frac{\mathrm{i}}{\hbar} \int_{0}^{\infty} \mathrm{d} t_{1} \theta\left(t_{1}\right) \exp \left(-\mathrm{i} \frac{\hat{L}}{\hbar}-\frac{\gamma}{\hbar}\right) t_{1}\right) \\
& \left.\times\left[\int \mathrm{d}^{3} \mathbf{r}^{\prime} \hat{\mathbf{P}}\left(\mathbf{r}^{\prime}\right) \cdot \mathbf{E}\left(\mathbf{r}^{\prime}, t-t_{1}\right)+\int \mathrm{d}^{3} \mathbf{r}^{\prime} \hat{\mathbf{M}}\left(\mathbf{r}^{\prime}\right) \cdot \mathbf{B}\left(\mathbf{r}^{\prime}, t-t_{1}\right), \rho^{(0)}\right]\right\} .
\end{aligned}
$$

Then the nonlocal response tensors $\alpha\left(\mathbf{r}, \mathbf{r}^{\prime}, t\right), \beta\left(\mathbf{r}, \mathbf{r}^{\prime}, t\right), \gamma\left(\mathbf{r}, \mathbf{r}^{\prime}, t\right)$ and $\kappa\left(\mathbf{r}, \mathbf{r}^{\prime}, t\right)$ are written as:

$$
\begin{aligned}
& \alpha\left(\mathbf{r}, \mathbf{r}^{\prime}, t\right)=\frac{\mathrm{i}}{\hbar} \operatorname{Tr}\left[\hat{\mathbf{P}}(\mathbf{r}) \theta(t) \exp \left(\left(-\mathrm{i} \frac{\hat{L}}{\hbar}-\frac{\gamma}{\hbar}\right) t\right)\left[\hat{\mathbf{P}}\left(\mathbf{r}^{\prime}\right), \rho^{(0)}\right]\right], \\
& \beta\left(\mathbf{r}, \mathbf{r}^{\prime}, t\right)=\frac{\mathrm{i}}{\hbar} \operatorname{Tr}\left[\hat{\mathbf{P}}(\mathbf{r}) \theta(t) \exp \left(\left(-\mathrm{i} \frac{\hat{L}}{\hbar}-\frac{\gamma}{\hbar}\right) t\right)\left[\hat{\mathbf{M}}\left(\mathbf{r}^{\prime}\right), \rho^{(0)}\right]\right], \\
& \gamma\left(\mathbf{r}, \mathbf{r}^{\prime}, t\right)=\frac{\mathrm{i}}{\hbar} \operatorname{Tr}\left[\hat{\mathbf{M}}(\mathbf{r}) \theta(t) \exp \left(\left(-\mathrm{i} \frac{\hat{L}}{\hbar}-\frac{\gamma}{\hbar}\right) t\right)\left[\hat{\mathbf{P}}\left(\mathbf{r}^{\prime}\right), \rho^{(0)}\right]\right], \\
& \kappa\left(\mathbf{r}, \mathbf{r}^{\prime}, t\right)=\frac{\mathrm{i}}{\hbar} \operatorname{Tr}\left[\hat{\mathbf{M}}(\mathbf{r}) \theta(t) \exp \left(\left(-\mathrm{i} \frac{\hat{L}}{\hbar}-\frac{\gamma}{\hbar}\right) t\right)\left[\hat{\mathbf{M}}\left(\mathbf{r}^{\prime}\right), \rho^{(0)}\right]\right] .
\end{aligned}
$$

These nonlocal tensors will be switched to the frequency domain after the Fourier transformation as

$$
\alpha\left(\mathbf{r}, \mathbf{r}^{\prime}, \omega\right)=-\operatorname{Tr}\left[\hat{\mathbf{P}}(\mathbf{r})(\hbar \omega-\hat{L}+\mathrm{i} \gamma)^{-1}\left[\hat{\mathbf{P}}\left(\mathbf{r}^{\prime}\right), \rho^{(0)}\right]\right],
$$




$$
\begin{aligned}
& \beta\left(\mathbf{r}, \mathbf{r}^{\prime}, \omega\right)=-\operatorname{Tr}\left[\hat{\mathbf{M}}(\mathbf{r})(\hbar \omega-\hat{L}+\mathrm{i} \gamma)^{-1}\left[\hat{\mathbf{P}}\left(\mathbf{r}^{\prime}\right), \rho^{(0)}\right]\right], \\
& \gamma\left(\mathbf{r}, \mathbf{r}^{\prime}, \omega\right)=-\operatorname{Tr}\left[\hat{\mathbf{P}}(\mathbf{r})(\hbar \omega-\hat{L}+\mathrm{i} \gamma)^{-1}\left[\hat{\mathbf{M}}\left(\mathbf{r}^{\prime}\right), \rho^{(0)}\right]\right], \\
& \kappa\left(\mathbf{r}, \mathbf{r}^{\prime}, \omega\right)=-\operatorname{Tr}\left[\hat{\mathbf{M}}(\mathbf{r})(\hbar \omega-\hat{L}+\mathrm{i} \gamma)^{-1}\left[\hat{\mathbf{M}}\left(\mathbf{r}^{\prime}\right), \rho^{(0)}\right]\right] .
\end{aligned}
$$

In TDHF equation, the discussion above is slightly modified due to the nonlinearity of the equation of motion in terms of $\rho$. Instead, equation of motion for linear response (Eq. (36)) is used and $\hat{L}$ is replaced by the linearized Liouville operator $L$ [49]

$$
L \rho^{(1)} \equiv\left[\delta h^{(1)}, \rho^{(0)}\right]+\left[h^{(0)}, \delta \rho^{(1)}\right] .
$$

Under the above replacement, Eqs. (A.22)-(A.25) hold. So far we have presented a methodology to calculate the response tensor of an arbitrary molecular system.

We do not invoke the dipole approximation. If the dipole approximation is employed, the rate of energy absorption at $\omega$ is

$$
A(\omega)=2 \omega \operatorname{Im}\left(\mathscr{E}^{*}(\omega) \cdot \alpha(\omega) \cdot \mathscr{E}(\omega)\right),
$$

which just depends on the tensor $\alpha=-\operatorname{Tr}\left[\hat{\mu}(\hbar \omega-\hat{L}+\mathrm{i} \gamma)^{-1}\left[\hat{\mu}, \rho^{(0)}\right]\right]$.

\section{References}

[1] K. Nakanishi, N. Berova, R.W. Woody, Circular Dichroism: Principles and Applications, VCH, New York, 1994.

[2] G.D. Fasman, Circular Dichroism and the Conformational Analysis of Biomolecules, Plenum Press, New York, 1996.

[3] A. Rodger, B. Nordén, Circular Dichroism and Linear Dichroism, Oxford, New York, 1997.

[4] N. Purdie, H.G. Brittain, Analytical Applications of Circular Dichroism, Elsevier, Amsterdam, 1994.

[5] R.K. Kondru, P. Wipf, D.N. Beratan, J. Am. Chem. Soc. 120 (1998) 2204.

[6] R.K. Kondru, P. Wipf, D.N. Beratan, Science 282 (1998) 2247.

[7] F. Furche, R. Ahlrichs, C. Wachsmann, E. Weber, A. Sobanski, F. Vögtle, S. Grimme, J. Am. Chem. Soc. 122 (2000) 1717.

[8] P. Bouř, J. Phys. Chem. A 103 (1999) 5099.

[9] J. Applequist, J. Chem. Phys. 71 (1979) 4332.

[10] P.M. Bayley, E.B. Nielsen, J.A. Schellman, J. Phys. Chem. 73 (1969) 228.

[11] H. DeVoe, J. Chem. Phys. 41 (1964) 393;

J. Chem. Phys. 43 (1965) 3199.

[12] W. Moffitt, A. Moscowitz, J. Chem. Phys. 30 (1959) 648.

[13] W. Rhodes, J. Chem. Phys. 53 (1970) 3650.

[14] T. Wagersreiter, S. Mukamel, J. Chem. Phys. 105 (1996) 7995.

[15] M.C. Manning, M. Illangasekare, R.W. Woody, Biophys. Chem. 31 (1988) 77.

[16] M.C. Manning, R.W. Woody, Biopolymers 31 (1991) 569.

[17] N.A. Besley, J.D. Hirst, J. Am. Chem. Soc. 121 (1999) 9636.

[18] N. Sreerama, R.W. Woody, J. Mol. Biol 242 (1994) 497.

[19] R.W. Woody, N. Sreerama, J. Chem. Phys. 111 (1999) 2844.

[20] K.A. Bode, J. Applequist, J. Phys. Chem. 100 (1996) 17825.

[21] K.A. Bode, J. Applequist, J. Phys. Chem. A 101 (1997) 9560.

[22] K.A. Bode, J. Applequist, Macromolecules 30 (1997) 2144.

[23] K.A. Bode, J. Applequist, J. Am. Chem. Soc. 120 (1998) 10938.

[24] J. Applequist, K.A. Bode, D.H. Appella, L.A. Christianson, S.H. Gellman, J. Am. Chem. Soc. 120 (1998) 4891.

[25] J. Applequist, J. Phys. Chem. A 102 (1998) 7723.

[26] J. Applequist, K.A. Bode, J. Phys. Chem. B 103 (1999) 1767.

[27] J.D. Hirst, J. Chem. Phys. 109 (1998) 782.

[28] S. Yokojima, G.H. Chen, Chem. Phys. Lett. 292 (1998) 379. 
[29] S. Yokojima, G.H. Chen, Phys. Rev. B 59 (1999) 7259.

[30] S. Yokojima, G.H. Chen, Chem. Phys. Lett. 300 (1999) 540.

[31] S. Yokojima, D.H. Zhou, G.H. Chen, Chem. Phys. Lett. 302 (1999) 495.

[32] W.Z. Liang, S. Yokojima, G.H. Chen, J. Chem. Phys. 110 (1999) 1844.

[33] W.Z. Liang, S. Yokojima, D.H. Zhou, G.H. Chen, J. Phys. Chem. A 104 (2000) 2445.

[34] W.Z. Liang, X.J. Wang, S. Yokojima, G.H. Chen, J. Am. Chem. Soc. 122 (2000) 11129.

[35] S. Yokojima, X.J. Wang, D.H. Zhou, G.H. Chen, J. Chem. Phys. 111 (1999) 10444.

[36] W.Z. Liang, S. Yokojima, G.H. Chen, J. Chem. Phys. 113 (2000) 1403.

[37] S. Yokojima, D.H. Zhou, G.H. Chen, Chem. Phys. Lett. 333 (2001) 397.

[38] P. Pariser, R.G. Parr, J. Chem. Phys. 21 (1953) 767;

J.A. Pople, Trans. Faraday Soc. 49 (1953) 1375.

[39] J. Del Bene, H.H. Jaffé, J. Chem. Phys. 48 (1968) 1807; J. Chem. Phys. 4050 (1968).

[40] J.J.P. Stewart, J. Comput. Chem. 10 (1989) 209.

[41] J.E. Ridley, M.C. Zerner, Theoret. Chim. Acta 32 (1973) 111; Theoret. Chim. Acta 42 (1976) 223.

[42] E.A. Power, S. Zienau, Philos. Trans. R. Soc. 251 (1959) 427.

[43] D.P. Craig, T. Thirunamachandran, Molecular Quantum Electrodynamics, Dover, Mineola NY, 1998.

[44] S. Mukamel, Principles of Nonlinear Optical Spectroscopy, Oxford, New York, 1995.

[45] J. Van Kranendonk, J.E. Sipe, in: E. Wolf (Ed.), Progress in Optics, XV, North-Holland, Amsterdam, 1977 , p. 245.

[46] L.D. Barron, Molecular Light Scattering and Optical Activity, Cambridge University Press, Cambridge, 1982.

[47] W.H. Press, B.P. Flannery, S.A. Teukolsky, W.T. Vetterling, Numerical Recipes in C, Cambridge University Press, New York, 1988.

[48] F. London, J. Phys. Radium 8 (1937) 397.

[49] A. Takahashi, S. Mukamel, J. Chem. Phys 100 (1994) 2366.

[50] A. Brown, C.M. Kemp, S.F. Mason, J. Chem. Soc. A 751 (1971).

[51] K. Palewska, H. Chojnacki, Mol. Cryst. Liq. Cryst. 229 (1993) 31. 